\title{
Índice de Adequação Idade-Anos de Escolaridade
}

ロ

Carlos Eduardo Moreno Sampaio

Vanessa Nespoli

Palavras-chave: escolaridade; ensino fundamental;

universalização do ensino fundamental; atendimento escolar; índicedeadequação idade-anos deescolaridade; fluxo escolar.

\section{Resumo}

O País atingiu um nível de acesso da população de 7 a 14 anos à escola praticamente universal - acima de 97\%; entretanto, o nível de escolaridade média da população de 15 anos ou mais édeapenas 6,7 anos. Esse aparente paradoxo éexplicado pela bai xa eficiência do sistema educacional brasilei ro em produzir concluintes, ou seja, se por um lado o acesso é quase universal, por outro, ai nda é bai xo o percentual daquel es que concluemo ensino fundamental, sobretudo na idade adequada. O objetivo deste artigo érefletir sobre o concei to de universal ização do ensino fundamental eapresentar uma nova proposta de indicador para avaliar não apenas o acesso a esse nível de ensino, mas também sua conclusão. Entendemos universal izar como a obtenção de oito anos de escolaridade e não, simplesmente, estar freqüentando a escola.

\section{Introdução}

Em um sistema de ensino de progressão continuada, independentemente da forma de organização (seriada ou em ciclos), como é o caso do ensino fundamental, é possível avaliar a escolaridade al cançada pel o al uno por meio da última etapa ou sé rie concluída com sucesso (aprovação). 0 aluno quetenha freqüentado duas séries ou etapas com aprovação terá dois anos de escolaridade; por outro lado, caso ele tenha freqüentado o ensino fundamental por três anos e obtido aprovação em apenas duas séries ou etapas, terá também dois anos de escolaridade.

Usando esseraciocínio, épossível identificar o número de anos de escolaridade ideal paraumacriançanaidadei supondo, obviamente, seu ingresso no sistemanaidade adequada e, assim, obtendo sucesso em suatrajetória escolar.
No caso brasileiro, espera-se que uma criança aos sete anos tenha ingressado no ensino fundamental (nível de ensino obrigatório). Desta forma, aos 8 anos, ela teria, pelo menos, um ano de escolaridade; isto é, teria freqüentado a primeira série ou etapa do ensino fundamental sendo aprovada e estando, portanto, apta a freqüentar a série ou etapa seguinte.

O indicador proposto tem por objetivo avaliar o nível deadequação da escolaridade obtida pelas crianças brasileiras considerando as explicações descritas acima.

O ideal seria aval iar a escol aridade das crianças a partir de oito anos de idade. No entanto, há situações em que o aluno ingressa no ensino fundamental aos sete anos ecompleta os oito duranteo ano letivo. Para não cometer nenhum equívoco, como, por exemplo, considerar quecriança deoito anos de idade não tenha obtido ainda a escolaridade adequada, o indicador proposto vai 
considerar o nível de escolaridade das crianças a partir de nove anos de idade. Assim, uma criança aos 9 anos, numa situação ideal, teria deter, pel o menos, um ano de escolaridade, e assim sucessivamente. O indicador poderá considerar diferentes coortes de idade. Avaliaremos aqui o Índice de Adequação Idade-Anos de Escolaridade para a coorte de 9 a 16 anos, tendo como foco o ensino fundamental (oito anos de escolaridade). Veja a Tabela 1, abaixo:

Tabela 1 - Idade e anos de escolaridade considerados adequados para o cálculo do Índice de Adequação Idade-Anos de Escolaridade

\begin{tabular}{|c|c|}
\hline Idade & $\begin{array}{r}\text { Anos de escolaridade } \\
\text { (ao menos) }\end{array}$ \\
\hline 9 anos & 1 \\
\hline 10 anos & 2 \\
\hline 11 anos & 3 \\
\hline 12 anos & 4 \\
\hline 13 anos & 5 \\
\hline 14 anos & 6 \\
\hline 15 anos & 7 \\
\hline 16 anos & 8 \\
\hline
\end{tabular}

O indicador apresenta dois objetivos básicos: aval iar o acesso e a progressão das crianças no ensino fundamental; ou seja, aos 9 anos a criança deveria ter, ao menos, um ano de escolaridade, aos 12 anos, não menos quequatro anos deescol aridade, de tal forma que, aos 16 anos, concluiria o ensino fundamental (oito anos deescolaridade). Isso só ocorrerá se a criança tiver acesso naidadeadequadaesucesso durante toda a sua trajetória escolar.

\section{Formulação matemática}

O objetivo do indicador é, portanto, avaliar a proporção decrianças queestão na situação ideal descrita acima; assim, o Índice de Adequação Idade-Anos de Escolaridade será expresso por:

$$
I A I A_{i j}=\frac{\sum_{k=9}^{16} P_{k}^{e_{k}}}{\sum_{k=9}^{16} P_{k}{ }^{\prime}}
$$

onde:

$P_{k}^{e_{k}}$ éa população na idadek com pelo menos $e^{k}$ anos deescolaridade;

$P_{k}$ éa população na idadek;

$$
\begin{aligned}
& e^{k}=\left\{\begin{array}{l}
1, \text { se } \mathrm{k}=9 ; \\
2, \text { se } \mathrm{k}=10 ; \\
\ldots \\
8, \text { se } \mathrm{k}=16
\end{array}\right. \\
& k \in[9,16] \\
& P_{k}^{e_{k}} \leq P_{k} \text { e, portanto, } \text { IAIA }_{i j} \in[0,1]
\end{aligned}
$$

\section{Análise dos resultados}

Como já foi dito anteriormente, hásitua ções em queo aluno ingressa no ensi no fundamental aos seteanosepodecompletar, duranteo ano letivo, oito anos. Assim, ao invés de analisarmos a escolaridade obtida pelas

\begin{tabular}{|c|c|c|c|c|c|c|c|c|c|c|}
\hline \multirow{2}{*}{$\begin{array}{c}\text { Região } \\
\text { Geográfica }\end{array}$} & \multicolumn{5}{|c|}{1996} & \multicolumn{5}{|c|}{2003} \\
\hline & $\begin{array}{l}7 \text { e } 8 \\
\text { anos }\end{array}$ & $\begin{array}{c}7 \text { a } 10 \\
\text { anos }\end{array}$ & $\begin{array}{c}11 \text { a } 14 \\
\text { anos }\end{array}$ & $\begin{array}{c}7 \text { a } 14 \\
\text { anos }\end{array}$ & $\begin{array}{c}7 \text { a } 16 \\
\text { anos }\end{array}$ & $\begin{array}{l}7 \text { e } 8 \\
\text { anos }\end{array}$ & $\begin{array}{c}7 \text { a } 10 \\
\text { anos }\end{array}$ & $\begin{array}{c}11 \text { a } 14 \\
\text { anos }\end{array}$ & $\begin{array}{c}7 \text { a } 14 \\
\text { anos }\end{array}$ & $\begin{array}{c}7 \text { a } 16 \\
\text { anos }\end{array}$ \\
\hline Brasil & 90,7 & 92,4 & 90,1 & 91,2 & 87,6 & 96,8 & 97,6 & 96,8 & 97,2 & 94,9 \\
\hline Norte & 87,7 & 90,6 & 92,5 & 91,6 & 89,3 & 95,1 & 96,3 & 95,3 & 95,8 & 93,8 \\
\hline Nordeste & 84,4 & 86,8 & 86,0 & 86,4 & 83,0 & 95,1 & 96,1 & 95,9 & 96,0 & 93,6 \\
\hline Sudeste & 94,6 & 95,8 & 92,6 & 94,1 & 90,5 & 98,1 & 98,6 & 97,7 & 98,1 & 96,0 \\
\hline Sul & 95,3 & 96,5 & 90,9 & 93,6 & 89,1 & 98,3 & 98,7 & 97,4 & 98,0 & 95,7 \\
\hline Centro-Oeste & 91,9 & 94,0 & 91,9 & 92,9 & 89,2 & 96,4 & 97,7 & 96,3 & 97,0 & 94,9 \\
\hline
\end{tabular}
crianças deseteeoito anos deidade, observaremossimplesmentea freqüênciaà escola.

Tabela 2 - Taxa de Frequência à Escola por faixa etária - 1996/2003

Fonte: IBGE - PNADs 1996 e 2003; Tabela elaborada pelo MEC/Inep.

Nota: Exclusive as pessoas da área rural de Rondônia, Acre, Amazonas, Roraima, Pará e Amapá. 
A Tabela 2 nos mostra duas informações rel evantes sob o foco abordado neste artigo. Nos últimos anos houve, de fato, um aumento significativo do acesso à escola no Brasil . Em todas as regiões geográficas esseindicador está acima de $95 \%$, em especial na faixa etária de 7 a 14 anos. Ainda assim há diferenças entre as regi ões brasileiras, mesmo que pequenas. Final mente, nas idades de sete e oito anos a freqüência à escola é praticamente de $97 \%$, o que garante a possi bilidade de esse mesmo contingenteobter, ao final de oito anos, a escolaridadeideal, ou seja, o ensino fundamental completo.

Os resultados do Índice de Adequação Idade-Anos deEscolaridadeapresentados na Tabela 3 mostram que, em 2003, 70\% da população de 9 a 16 anos de idade conseguiram al cançar a escol ari dadeadequada a essa faixa etária. Em 1996, menos da metade dessa população possuía a escolaridade adequada, revelando um grande avanço, quando comparado ao índice de2003. Apesar disso, há, ainda, uma grande lacuna que precisa ser preenchida nos próximos anos.

Tabela 3 - Índice de Adequação I dade-Anos de Escolaridade por idade, segundo o ano - 1996/2003

\begin{tabular}{|c|c|c|c|c|c|c|c|c|c|}
\hline \multirow{2}{*}{$\begin{array}{c}\text { Ano/Região } \\
\text { Geográfica }\end{array}$} & \multicolumn{9}{|c|}{ Idade } \\
\hline & $\begin{array}{c}9 \text { a } 16 \\
\text { anos }\end{array}$ & 9 anos & 10 anos & 11 anos & 12 anos & 13 anos & 14 anos & 15 anos & 16 anos \\
\hline \multicolumn{10}{|c|}{1996} \\
\hline Brasil & 0,49 & 0,73 & 0,66 & 0,57 & 0,53 & 0,46 & 0,39 & 0,35 & 0,29 \\
\hline Norte & 0,39 & 0,67 & 0,55 & 0,48 & 0,43 & 0,35 & 0,26 & 0,23 & 0,20 \\
\hline Nordeste & 0,28 & 0,49 & 0,41 & 0,32 & 0,26 & 0,23 & 0,19 & 0,18 & 0,14 \\
\hline Sudeste & 0,63 & 0,89 & 0,80 & 0,72 & 0,69 & 0,59 & 0,52 & 0,46 & 0,39 \\
\hline Sul & 0,66 & 0,89 & 0,85 & 0,79 & 0,73 & 0,62 & 0,54 & 0,49 & 0,41 \\
\hline Centro-Oeste & 0,52 & 0,79 & 0,72 & 0,63 & 0,58 & 0,48 & 0,38 & 0,33 & 0,27 \\
\hline \multicolumn{10}{|c|}{2003} \\
\hline Brasil & 0,70 & 0,89 & 0,84 & 0,78 & 0,74 & 0,66 & 0,62 & 0,57 & 0,51 \\
\hline Norte & 0,59 & 0,85 & 0,72 & 0,67 & 0,65 & 0,51 & 0,50 & 0,44 & 0,36 \\
\hline Nordeste & 0,52 & 0,81 & 0,73 & 0,62 & 0,56 & 0,45 & 0,40 & 0,36 & 0,29 \\
\hline Sudeste & 0,81 & 0,94 & 0,91 & 0,89 & 0,85 & 0,78 & 0,77 & 0,72 & 0,67 \\
\hline Sul & 0,81 & 0,95 & 0,93 & 0,88 & 0,86 & 0,81 & 0,72 & 0,69 & 0,63 \\
\hline Centro-Oeste & 0,74 & 0,92 & 0,87 & 0,83 & 0,78 & 0,74 & 0,67 & 0,58 & 0,52 \\
\hline
\end{tabular}

Fonte: IBGE - PNADs 1996 e 2003; Tabela elaborada pelo MEC/Inep.

Nota: Exclusive as pessoas da área rural de Rondônia, Acre, Amazonas, Roraima, Pará e Amapá.

Outro aspecto rel evanteéque, à medidaqueaidadeavança, háuma perdadeprodutividade no sistema educacional, pois se deum lado temos umaalta taxa defreqüência à escola, por outro, oĺndicedeAdequa ção Idade-Anos de Escolaridade diminui progressivamente. Bastaobservar queaos 16 anos deidade pouco mais de $50 \%$ da população obtevea escol aridadeideal. Ainda persistem as desi gual dades regionais. $\mathrm{Na} \mathrm{Re}$ gi ão Nordeste, $52 \%$ da população de 9 a 16 anos al cançaram a escolaridadeadequadaà sua idade, entretanto, menos de $30 \%$, aos
16 anos de idade, conquistou o ensino fundamental completo. $E$, mesmo nas regiões mais desenvol vidas economi camente, o Índice de A dequação Idade-Anos de Escol aridade aos 16 anos não ul trapassa 70\%.

Considerando o ensino fundamental em duas fases (1a a 4a e 5a a 8a séries), o Índice de Adequação Idade-A nos de Escolaridade podeser aval iado levando em consi deração os grupos etários de 9 a 12 e de 13 a 16 anos. Obviamente, os resultados da Tabela 4 revelam situação semel hante à descrita anteriormente. 
Tabela 4 - Índice de Adequação Idade-Anos de Escolaridade por faixa etária - 1996/2003

\begin{tabular}{|l|r|r|r|r|r|r|}
\hline \multicolumn{1}{|c|}{$\begin{array}{c}\text { Região } \\
\text { Geográfica }\end{array}$} & \multicolumn{1}{|c|}{$\begin{array}{c}\text { 1996 } \\
\text { 9 a 16 } \\
\text { anos }\end{array}$} & $\begin{array}{c}\text { 9 a 12 } \\
\text { anos }\end{array}$ & $\begin{array}{c}\text { 13 a 16 } \\
\text { anos }\end{array}$ & $\begin{array}{c}\text { 9 a 16 } \\
\text { anos }\end{array}$ & $\begin{array}{c}\text { 9 a 12 } \\
\text { anos }\end{array}$ & $\begin{array}{c}\text { 13 a 16 } \\
\text { anos }\end{array}$ \\
\hline Brasil & $\mathbf{0 , 4 9}$ & $\mathbf{0 , 6 2}$ & $\mathbf{0 , 3 7}$ & $\mathbf{0 , 7 0}$ & $\mathbf{0 , 8 1}$ & $\mathbf{0 , 5 9}$ \\
\hline Norte & 0,39 & 0,53 & 0,26 & 0,59 & 0,72 & 0,45 \\
\hline Nordeste & 0,28 & 0,37 & 0,18 & 0,52 & 0,68 & 0,37 \\
\hline Sudeste & 0,63 & 0,77 & 0,49 & 0,81 & 0,90 & 0,73 \\
\hline Sul & 0,66 & 0,81 & 0,52 & 0,81 & 0,91 & 0,71 \\
\hline Centro-Oeste & 0,52 & 0,68 & 0,36 & 0,74 & 0,86 & 0,62 \\
\hline
\end{tabular}

Fonte: IBGE - PNADs 1996 e 2003; Tabela elaborada pelo MEC/Inep.

Nota: Exclusive as pessoas da área rural de Rondônia, Acre, Amazonas, Roraima, Pará e Amapá.

O Gráfico 1 mostrao ÍndicedeAdequação Idade-Anos deEscolaridade por Unidade da Federação, e constata-se situação reveladapelamaioria dosindicadores sociais; ou seja, abaixo damédianacional sóseencontram Estados das Regiões NorteeNordeste.



Gráfico 1 - Índice de Adequação Idade-Anos de Escolaridade na faixa etária de 9 a 16 anos por Unidade da Federação - 1996/2003

Fonte: IBGE - PNADs 1996 e 2003; elaborado pelo MEC/Inep.

Nota: Exclusive as pessoas da área rural de Rondônia, Acre, Amazonas, Roraima, Pará e Amapá.

Finalmente, o Gráfico 1 revela que o Estado brasileiro de melhor desempenho possui um Índice de Adequação IdadeAnos de Escolaridade duas vezes maior que o Estado de pior resultado.

\section{Conclusão}

Como vimos, no Brasil, as crianças têm acesso à escola na idade adequada, mas, infel izmente, grande parcela não consegue concluir a escolaridadeobrigatória no tempo adequado. Isso nos revela uma baixíssima produtividade dos sistemas de ensino, com grandes disparidades regionais.

Situações bastante distintas e preocupantes são ilustradas pelo Gráfico 2, em que se observam dois dos indicadores de produtividade de Fluxo Escol ar ${ }^{1}$ (Taxa Média Esperada de Conclusão e Tempo Mé dio Esperado para Conclusão). O Estado

\footnotetext{
Klein, Ruben. Produção eutilizacão deindicadoreseducacionais: metodologia decál culo de indicadores defluxo escolar da educação básica. Revista Brasileira de Estudos Pedagógicos, Brasília, v. 84, n. 206/207/208, p. 107-157, jan./dez. 2003.
} 
demenor TaxaEsperadadeConclusãoé, também, aquel eem queos alunos permanecem mais tempo para obter a escolaridade obrigatória, confirmando as conclusões obtidas peloĺndice deAdequação Idade-Anos de Escolaridade.

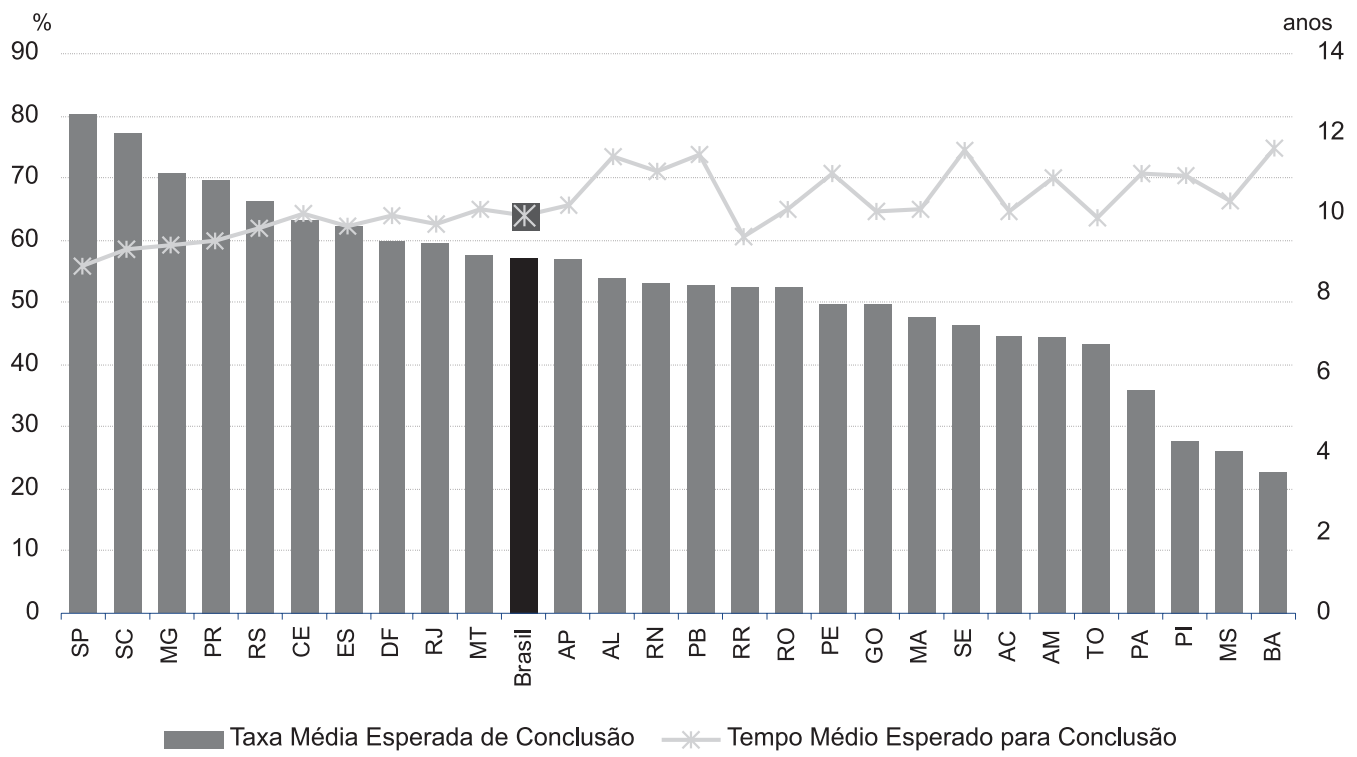

\section{Gráfico 2 - Ensino Fundamental - Taxa Média Esperada de Conclusão e Tempo Médio Esperado para Conclusão - 2002/2003}

Fonte: MEC/Inep

No Brasil, a Taxa Média Esperada de Conclusão do Ensino Fundamental é de $57,1 \%$, e os al unos que conseguem essa façanha gastam, em média, dez anos, ou seja, dois anos mais que o necessário. As razões são muitas e extrapolam os objetivos deste artigo, mas não poderíamos deixar demencionar a qualidade da educação, sobretudo a educação oferecidaà parcela da população quebusca a escola pública. Esta escola que desejamos precisa ser capaz deajudar a criança que freqüenta seus bancos a concluir seus estudos. Desta forma, cabem as reflexões: - O que ocorre com essa criança que freqüenta escola e não consegue o nível de escolaridadeque poderia? - Que papel tem a escola, a famíliae os gestores educacionais sobre esse desempenho tão bai xo?

Outros indicadores do Inep mostram que a rede privada de ensino tem comportamento absolutamente distinto do observado na rede pública. Outro aspecto a ser considerado, não menos importante, equetambém transcende aos objetivos do artigo, é que o País gasta mais do que deveria para produzir um concluinte do ensino fundamental. Não queremos dizer, entretanto, que os recursos destinados à educação sejam adequados. Seguramente a ampliação dos recursos deve ser fator decisivo para a mel horia da qual idade que estamos defendendo aqui .

Concluímos este artigo com uma visão otimista e destacamos, no Gráfico 3, as 27 unidades da Federação ordenadas de acordo com o avanço obtido no Índice de Adequação I dade-Anos de Escolaridadeem relação ao que poderia ter sido mel horado no período de 1996 a 2003.

No Gráfico 3, cabe o destaque a São Paulo, Estado demel hor desempenho, eaos Estados do Ceará e Tocantins, que, apesar de apresentarem um Índice de A dequação Idade-Anos de Escolaridadeabaixo da média nacional , empreenderam esforços superiores à média brasileira. 


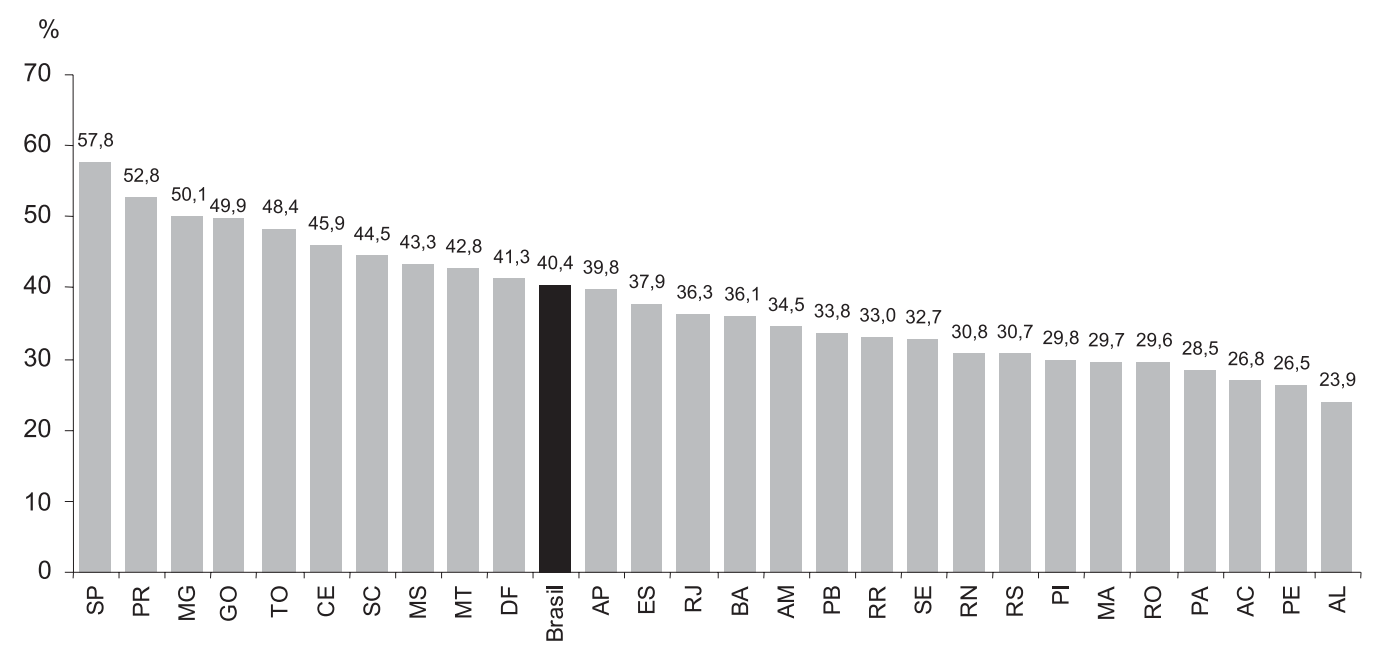

\section{Gráfico 3 - Percentual de Melhoria no Índice de Adequação Idade-Anos de Escolaridade na faixa etária de 9 a 16 anos, em relação ao que cada Unidade da Federação poderia mel horar* - 1996/2003}

Fonte: IBGE - PNADs 1996 e 2003; Elaborado por MEC/Inep.

Notas: Exclusive as pessoas da área rural de Rondônia, Acre, Amazonas, Roraima, Pará e Amapá.

$(*)$

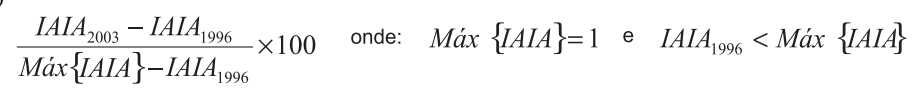

Carlos Eduardo M oreno Sampaio, mestre em Estatística e Métodos Quantitativos pela Universidade de Brasília (UnB), écoordenador-geral do Sistema Integrado del nformações Educacionais (SIEd) da Diretoria de Tratamento eDissemi nação de Informações Educacionais (DTDIE/Inep/MEC).

Vanessa Nespoli, especial ista em Estatística pela Universi dade de Brasília (UnB), é consultora da Coordenação-Geral doSIEd da DTDIE/Inep/MEC.

\section{Abstract Index of age-grade gap}

Brazil provides nearly universal access to school for the population aged 7 to 14 above $97 \%$; however, the average schooling level of the population aged 15 or more is only 6.7 years. This apparent paradox is explained by the low efficiency of the Brazilian educational system in producing graduates; in other words, if on one hand access is almost universal, on the other, the percentage of those who conclude basic education is still low, mainly in the proper age. The objective of this article is to reflect on the concept of universalization of basic education and to present a new proposal of indicators not only to evaluate access to this schooling level, but also to its conclusion. We understand universalization as concluding basic education in eight years, not only as attending school.

Keywords: schooling, primary education, universalization of primary education, school attendance, age-years schooling adequation index, school curriculum.

Recebido em 2 de maio de 2005. 